\title{
Claus Janew
}

\section{Alternating Consciousness}

\author{
From Perception to Infinities \\ and Back to Free Will
}

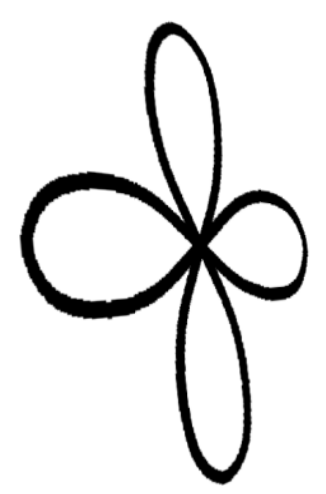

Can we trace back consciousness, reality, awareness, and free will to a single basic structure without giving up any of them? Can the universe exist in both real and individual ways without being composed of both? This dialogue founds consciousness and freedom of choice on the basis of a new reality concept that also includes the infinite as far as we understand it. Just the simplest distinction contains consciousness. It is not static, but a constant alternation of perspectives. From its entirety and movement, however, there arises a freedom of choice being more than reinterpreted necessity and unpredictability. Although decisions ultimately involve the whole universe, they are also free in varying degrees here and now. The unity and openness of the infinite enables the individual to be creative while this creativity directly and indirectly enters into all other individuals without impeding them. A contrary impression originates only in a narrowed awareness. But even the most conscious and free awareness can neither anticipate all decisions nor extinguish individuality. Their creativity is secured.

This e-book is based on my work which was originally completed in German in 2013 and published in 2014 in the Journal of Consciousness Exploration \& Research. Get the print edition with an introductory article from Amazon.

\section{https://free-will.de/}




\section{Day 1: What is a consciousness unit?}

Mr. Janew, you claim to have discovered a basic structure of consciousness. What do you mean by that?

Well, something on which everything else is based on must be as simple as possible. Only then it can be contained in everything else and determine structure and action there. In part, this something is even well-known.

Oh? What is it then?

Alternation.

You mean change? Like Heraclitus could not step into the same river twice?

Continuous change is a special form of alternation, with many intermediate steps, which we cannot easily resolve. But if Heraclitus briefly closes and re-opens his eyes, he has changed his point of view more clearly.

Okay, forget Heraclitus. We have an alternation. At which point does the consciousness come into play?

It is already in the play, because alternation is already consciousness, even in its simplest form. Not only because we observe it, but because it contains something that we have not taken seriously up to now: The central point. Let us take the simplest conceivable alternation between two whatever, here represented by alternatively flashing squares:

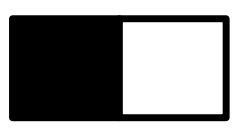

They must not flash side by side; they can substitute for each other. We need neither space nor time for this. It is only an alternation of priority. However, each square is only measured against the other, or there would be none of them. This means that each exists only in the alternation. The alternation is an entirety. And an entirety has a central point.

Okay, and where is the consciousness?

Look again. The squares are for illustrative purposes only. They could be anything that is in any way differentiated, demarcated from each other. This difference has an infinitely small center, a third thing, so to speak, which also stands and falls with the alternation like the alternating sides. Only such an entirety can work. Everything else falls apart.

And where now is the consciousness?

Consciousness is just this holistic perception. This perception is intuitive and logical; it is experienced directly, without necessary intermediate stages. And, nevertheless, it can be broken down, 
extended, and understood. It is self-referential and ubiquitous. It reaches to the infinitely small and to the infinitely big, into the simple and into the complex. It is the most general of our perceptions; and more than perception we do not have. What else do you want to assign to a consciousness?

Hmm ... So we could also say, conversely: We take our most natural perception and look at its least structure, and this is that ...

... infinitesimality structure. Yes, exactly. For simplicity's sake we can call it "i-structure."

I-Structure therefore is consciousness?

Yes.

Isn't something still missing here? Feelings, for example? Or perception of a color, a tone?

As everybody knows, all these are oscillations, therefore different forms of alternations, which we perceive holistically. Now, though, we must be careful:

What I have just described is the absolute minimum, a consciousness unit. Such a minimum can't differ from other minima without already forming a larger structure with them. This means vice versa: Each consciousness unit can only exist within a larger consciousness by which it is defined.

Doesn't this mean chasing one's own tail? Shouldn't the units build up a larger consciousness instead of being determined by it?

One presupposes the other. The larger consciousness needs elements of its structure, and the basic consciousness needs a larger structure in which it takes a characteristic position. In addition, of course, we always start with our consciousness that should not be so basic.

What is the difference then between a consciousness unit and an elementary particle if we assume that the latter is really elementary?

Just this we cannot assume. Up to now, we have still disassembled every particle after a short time if it has not done this by itself. But if a real elementary particle would exist, it could only interact by entering a larger relationship, and so it had the same problem as the consciousness unit. It loses its originality; it only exists in the relationship.

So, only the particular starting point of the perception is original ...?

Exactly.

However, this perception is not so i-structured, is it? We see surfaces, bodies, et cetera.

It is! Since we always perceive only entireties, every change of a perception is a change of the entirety. So if you go one step to the side, your holistic perception, let's say of a body, has changed completely. In order to notice the change you must compare to the perception of the previous entirety, and so you have the same switching back and forth.

But there are many intermediate stages here. I perceive, after all, a uniform change of my field of vision. 
Right. This, though, does not change the basic fact of the holistic alternation. Whether it takes place continuously or by leaps is of secondary importance. You can even say all sides of the alternation are always also immediately linked to each other, since the only necessary and always existing transition point is the infinitesimal center between them. An infinitely small transition yet takes place immediately.

Why do we need this transition if it is not really there?

It is there and not there all at once. For this reason, it is infinitely small and not simply zero. On the one hand, it is determined exactly as a center; on the other hand, it is empty. We need to have it as exactly that, as a nothing with a concrete meaning. As a concrete nothing.

To approach this point infinitely, nevertheless, it requires a transition to it. Now you say, this transition is actually not needed because the alternation between the sides occurs immediately.

This is due to the fact that we have nothing but the alternation. Each intermediate stage toward the center would also be the goal of an alternation. Thus, we can approximate the central point via many intermediate alternations, but strictly speaking each center remains immediately accessible. However, because it can be circumscribed arbitrarily closely, it is also approximated. It is both infinitely small as well as zero.

A consciousness unit doesn't have meaning at all if it doesn't transition to a structure. It has only meaning within this structure as their almost infinitesimal center. What I have described as two alternating whatever are just such structures. An alternation between nothing cannot be, of course.

But alternation as such can be?

Yes. Because everything alternates, and we cannot go beyond alternation as such. It forms the apparently static structures of the world, which I call therefore "quasi-static." Back-and-forthmovements, rotations, alternations in all possible forms.

So, in a sense the world becomes ethereal. There is nothing solid, no minimum size, nothing that can be called truly material. How do you fit the quantum theory in here? In it there is at least Planck's quantum of action as the smallest arithmetic unit.

This quantum, too, is being questioned yet. As well as the constancy of the "fundamental constants." An absolute quantity is simply not thought through. Any limit can be exceeded, because this limit is defined by its momentary exceedance. Try it out!

Nevertheless, quantum physics describes completely different relations, entangled states of so called particles: Nonlocal correlations, probability waves, etc.

I am inclined to say such unmediated connections over long distances point in the direction which I have already described. We must see, however, that the declaration of an unmediated link is only possible outside of the immediacy. We must walk across to the other particle quite normally to compare its state to "our" particle. Their immediate connection is a conclusion from a non-immediate connection. Anyway, the immediacy plays a more significant role here than in our everyday 
experience. You can hardly abandon it, because obviously it is structurally deeper rooted. Especially their probability character suggests that.

This brings me to another question: In How Consciousness Creates Reality you give to the central point far more meaning. You see in it, so to speak, the continuum of the world compacted. How does this fit here?

Well, a consciousness unit as the absolutely smallest before zero must alternate at infinite speed, because there is no space for delays. However, as soon as we go beyond this unit, better said return from its derivation, the speed can decrease. And with it two manners of perception of the alternation start to differ: The quasi-static and the dynamic.

The quasi-static perspective you have already indicated ...

Yes, it is the formation of seemingly static objects from the alternation of the perspective ...

... which in turn results from other smaller or larger alternations of perspectives.

Or from remembered and anticipated, mental and sensory, dreamed and awake-conscious experiences.

That's a lot of perspectives, considering what the world all consists of!

Thus it is. And that's why we cannot follow up them all by our limited consciousness. We always move within a relatively small frame and then within the next, and so on, keeping the respectively others in the back of our mind as a potential. We can restore them largely or at least think of them as restorable, but we do not lose sight of the movement, of the alternation. This is the dynamic manner of perception. I call it awareness.

Is the aware conscious?

When we alternate into something and back again, both cannot be fully conscious together at any moment. Nevertheless, we must remain aware of the other side, otherwise the alternation would disappear, too. We are aware of the potential for restoring that side.

But isn't this a contradiction in itself? The goal of our alternation is not conscious, only the potential. And, on the other hand, does the alternation consist of both sides equally?

We must simply understand that we have only alternation as such. It includes both sides, but with alternating priority. There is no pause in which only one side exists or both sides exist at once. So the alternation is potential in some sense.

Why only in some sense?

Because the potential again seems to exist as such; like a quasi-static object, which alternation movement we are not conscious of any more. But we have only alternation as such. If we do not always want to get stuck again, we must get used to looking at it as nothing else than what it is. We cannot condense it to a static object and complain then about contradictions! 
On the other hand, a quasi-static object is but somehow static or not?

No, just only quasi.

\section{Because we do not look closely?}

Yes, because we are not able to do it. As soon as we remove from a consciousness unit or from All That Is to be discussed later we have a restricted speed of alternation. That is we can no longer be perfectly accurate, no longer apprehend everything, but must make approximations. We condense seemingly static objects. The alternation movement is being largely suppressed.

How should I imagine this condensing?

Look at the simple example from earlier. Now we have a distance between the alternating sides:

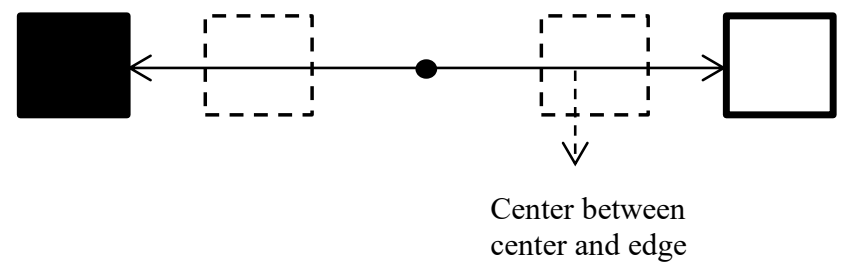

So, there are many intermediate steps, as you said. Accordingly, there are also many intermediate centers depending on between which steps alternation is happening. An overall center exists anyway. Now we can even alternate between this center and the edges resulting in new centers, and so on. The infinitesimality structure does justice to its name quite more clearly.

But I see no condensate.

Don't you? And I've painted the center even suspiciously large! Let us expand the whole a little bit more to a rotation of the sides:

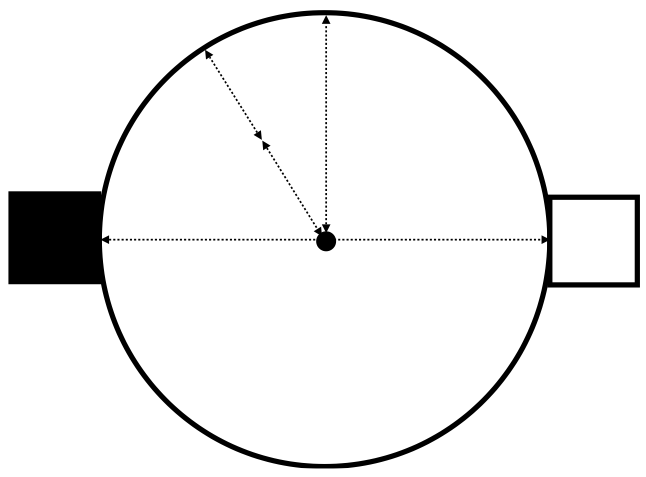

Do you see it now?

Hmm ... You mean the whole as such? 


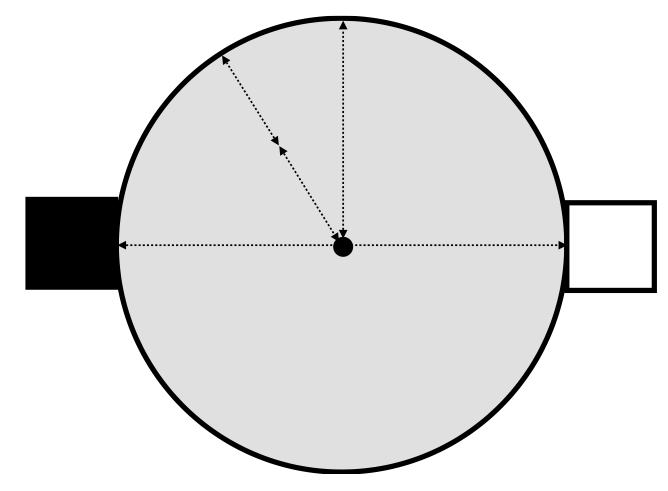

Not only that. The whole as such is relatively stable by the repetition of the alternation, the interdependence of the sides. But its stability is mainly symbolized by the center, because it moves the least. However, since the whole is extended its most representative central area forms around the central point:

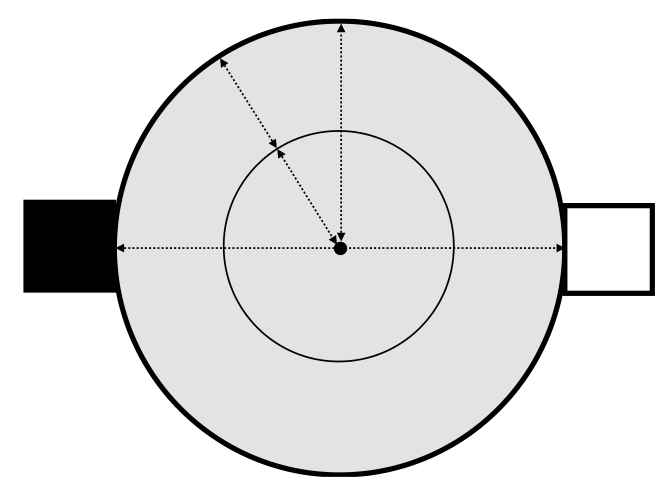

Where exactly that is does not depend only on the change of the movement ratio in the area between central point and edge, but also on the importance of the cohesion. For this particular central point applies only to exactly this entirety. It is related to the latter most strongly.

I understand. The central point is defined only in relation to the entirety.

Exactly. So, the more important the unity of the whole is compared to its differences, the closer the most representative area condenses at the center. Like this: 


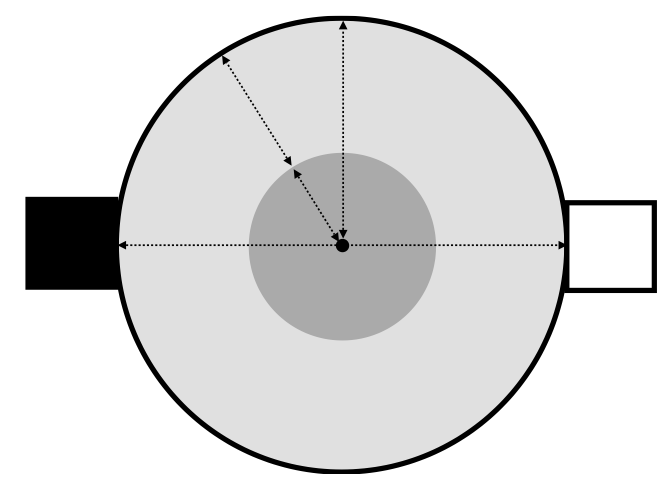

Of space and time we talk, by the way, only because we have got used to it. Actually, varying dream scenes, melodies, or whatever can also circumscribe a center; make feel an entirety, which condenses toward this core.

Well, I see, or I rather sense the condensate. What is the quasi-static object here? The condensate or the entirety?

Strictly speaking, the condensate. Since if we follow more the outsides it becomes quite dynamic, it resolves into alternating viewpoints. However, we can of course also look at the entirety as such from different perspectives and assign to it the role of the object, and so on.

So we solidify our imagination ... Anyway, I think you have described alternation and entirety as if they were self-contained. But in the world, indeed, everything is connected. How then the link to other alternation structures does come about?

We could also ask the other way around first. Why the sides do recur at all? Why there are turning points of the movement or change?

Okay, why?

Because, otherwise, there would be no alternation.

Aha. It's already a bit late, but now you should really answer your own question.

Oh, Okay. So, why turning points? They are one side of the alternation, and so, they appear as outposts in need for some impulse for a return. But you can also invert the alternation in some sense and consider both sides together as the center, which is circumscribed by the alternation towards it and back from it.

I think I have a knot somewhere ...

The operation is not symmetrical, but it shows that both sides can exist only together. They are a split center, split by the alternation. Beyond that is nothing. 
Except other alternations ... Wait a minute. Didn't you say every limit can be exceeded? Then, there must be something out there, anyway!

And now we come to the question of openness.

\section{Day 2: Choices everywhere}

On the openness of alternation it came back to my mind today that, actually, a consciousness unit is an abstraction from a larger context. So, it cannot be complete at all, and thus, no other wholeness composed of consciousness units. Or?

Yes, and no. A self-contained unit could not exist for anything else, so far I agree. However, we must allow these extremes as you will notice.

I'm all ears.

Let us continue with the rotation, because it is more descriptive. Instead, we could take a to-and-froalternation or something more complicated as well.

The path from one side to the other is not as clear-cut as it looks in the drawings. In reality it furcates continuously, because otherwise it would mean an uncrossable limit. But such a limit is inadmissible at every moment, because it is not consistently definable.

Why then does anything return to the starting point if there are so many other options?

I have a shocking answer. Yet, first of all, I ask you this: What would be left longest if all progression routes, open and closed, were used?

Hmm ... The closed ones?

Precisely. And if the open ones were totally open, they would not exist for a single moment. Since who should perceive an entirety? On the other hand: Total self-containment would change not the least; so it would be not connectable, not perceivable.

Okay, how do we get out of this dilemma?

No suggestion?

Consciousness unit?

Hit.

But how?

As you know, a consciousness unit is also an entirety, while its sides are alternating immediately in zero time and hence as immediately into the central point and out of it. It is an infinitely small alternation structure, but also more than zero. 
Is it what is called an infinitesimal in the non-standard mathematics, a number infinitely close to zero?

Not really, because these numbers in the non-standard analysis again are treated only as an object. In contrast, a consciousness unit is constantly flickering. It is alternating between precisely zero and infinitesimal sides.

Ah! And thus, self-containment and openness are unified! By lying infinitely close to each other.

Not just! But by alternating infinitely fast to each other! This is another thing than an asymptotic approach in which they meet in the infinitely small. I mean openness and self-containment at the same moment!

Without contradiction to each other ...

Without unhealthy contradiction. Since the "contradiction" of which we speak here is omnipresent, the basis of our world. It has no opposite to be preferred, because the latter would disappear at the same moment.

Didn't they call it earlier a dialectical contradiction? Hegel ...

Hegel didn't call it that, though, he realized the unity of existence and nonexistence, or as he understood it, of being and nothingness. Not only because one needs the other to be defined, but because one is constantly transitioning to the other. Everything is always becoming.

And this is something different?

Hegel has only gone halfway. He believed to have proved the necessity of the world process, but he has already assumed it. Becoming is not alternation. In the becoming there is no furcation, this can only be added from outside. In the alternation, however, furcation is built in.

\section{Between openness and self-containment, I understand.}

Also between different open paths, as we shall see. But let's get back first to the unity of openness and self-containment. This unity is not lukewarm or vague, although it can be if we dilute it to an approximation. Instead, it goes to the most precise. There is even no separation between selfcontainment and openness in the last consequence, so that objects always find connection to other objects.

Otherwise, we could not have derived the consciousness units from them as well.

Exactly.

\section{What about the extreme case of total self-containment that you mentioned?}

It must be there as well as the extreme case of total openness and all other extreme cases. Since each side of the alternation is being actually reached, as well as the central point, although only for an infinitely small moment.

This is why the unity with the other side is possible at all. Slowly I understand. 
I am pleased.

Although I am not shocked.

Huh?

You have promised me a shock.

Oh, yes. Openness in itself is not everything. If the door is already open, we can turn into different directions as well. Otherwise, we would have a self-containment of its own kind again.

The containment of the direction.

No roundabout, but no other alternative, yes. That is we are again at a furcation.

What do we do now?

We choose.

$O h !$

Are you shocked?

Maybe later.

The alternation between two or more sides after all is nothing else than weighing up alternatives. The only thing we are forced to is keep moving. For alternation is unavoidable on penalty of our elimination. This means, we are always in a situation of decision-making.

... About the way forward. You have to let this melt in your mouth.

As you wish. Anyway, the direction of further alternation of further movement is indeterminate.

Here I have to digress: Motion is asymmetrical, open, as you know. Nevertheless, it can only exist in the change-wise perception of its previous segments; otherwise, it dissolves more rapidly than we can say "Fzz." It would not even have a direction, which in turn only exists in the change-wise comparison with its alternatives. It would be the extreme case of total openness and therefore of being structureless.

Again, I do not understand "direction" as spatiotemporal in the first place, but as a direction from one priority to the other. If we can draw it in the space-time diagram, Okay. However, also associations, for example, have directions from the important to the not yet important.

Your cats, are they brother and sister?

Are you still with me?

Yes, sorry. Do you speak of a spiral?

Spiral?

Yes, a spiral movement. A back-and-forth-switch between moments while the whole is moving forward results in a spiral pulled apart to the side. 
Only superficially, though. This spiral is rather a manifestation of an i-structure, of a complete alternation of moment points, which still jumps forward immediately. An i-structured spiral, if you like.

Can you give an example?

Of course. We were choosing, do you remember? We are alternating between alternatives of our further movement, of our potential. One of them we have to take. Let's say, either a new way or an old one; we are choosing between an open and a self-contained continuation. Only the indetermination and the choosing as such are determined.

Thus, we are also alternating between this indetermination and our determination to choose. That is we are circumscribing a center between the alternatives as well as between the alternatives and the urge to choose.

And by this a center between these both sides ...

Yes.

And centers between this center and the others.

And so on.

\section{This is infinitesimality structure!}

All sides of the alternation are identified with each other at some point and at some other even with their distinction.

What normally doesn't work according to conventional understanding ...

... but as we have seen, is the basis of our world down to the smallest conceivable unit.

Can't we just say, the sides meet in the middle and one of them is being chosen?

We can say much and reveal nothing. Because in this way we cannot explain choosing, only mechanical continuations and chances. By such a merging we would disregard the necessity to distinguish things, too. We had merely flowing mush.

By contrast, what you have explained leads to a free choice?

Yes, because the infinitesimal unity of determination and indetermination is not annullable and not reducible to one side. Free choice, not chance, is the only interpretation that remains.

This is based, as far I can see, on the need to alternate rather than simply continuing.

Only alternation is distinction and unity at once. This alternation, however, can progress to other alternations. It will do this at some point not to lose touch with the world or, better said, because the alternatives are too tempting to forever decide against them, though it must not.

But I do. Can we take a short break?

Sure. In the meantime, I succumb again to the temptations of art. 

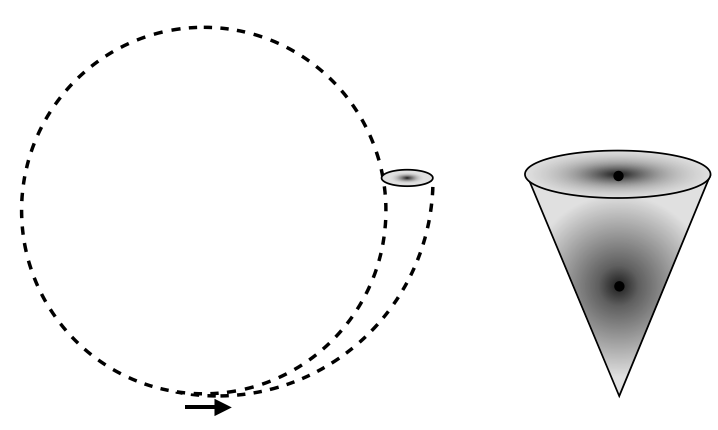

Alright, so we choose constantly between old and new way, since we always consider the new more or less. With it, self-containment and openness form an i-structured unity. If we always moderately decide for the new, we obtain the approximation of a spiral.

Let me think... If we describe the situation once again by using squares, we now have an alternation between three instead of two sides, and the third one stands for a new way.

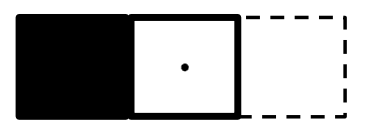

More specifically, it stands for the possibility of a new way. We alternate with a potential as such, that is to say without realizing it immediately. This is, in fact, an additional alternation. However, it is the normal case, which we have simplified yesterday, just almost up to a self-containment. If we now, like before at the door, open the direction of the continuation as well, we get even more alternatives of alternation:

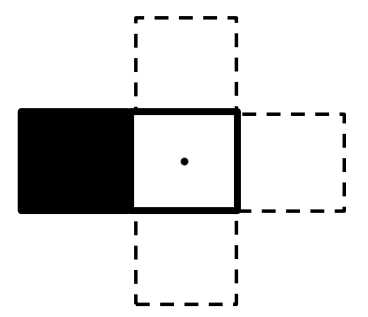

And because finally everything opens -it makes its potential conscious-decisions are to be made constantly. Whether an alternation moves on or not, it is always a more or less free choice of its consciousness!

You're so quiet.

Hmm ... The continuation is neither a real spiral nor a real jump, but a decision for one or the other as well? 
Well recognized.

And since we have everywhere alternation equals i-structure equals consciousness, and everything is more or less open, everything is freely chosen to the corresponding extent.

You got it!

\section{Day 3: Awareness in alternation}

If I sum up the last two days, then consciousness is omnipresent and due to its structure freedom of choice is just as omnipresent.

Right. The urge to change the situation, the i-structure, and the ultimate identity of self-containment and openness result in a permanent choice of the further path. These three factors are basically one and the same.

\section{Consciousness.}

I-structure, yes.

Or awareness?

This, too, is essentially the same. We have already discussed that every side is always potential, that there is only alternation as such. If we think to perceive two sides at once, strictly speaking we deceive ourselves. We lift them out of the broader alternation by turning them crosswise and thereby seemingly slowing down their alternation.

\section{Seemingly?}

I'll come back to this. By the said turning we generate an approximation around the center of the sides, call it "object" and forget its origin and the details, which we cannot resolve now anymore. The sides themselves are also formed in such a way and so on, because we can hardly do without crosswise things. Only a quasi-static consciousness can seemingly exist longer than zero "seconds" and has "time" to become aware of something.

Man oh man. Awareness must therefore be conscious?

Yes, conscious or subconscious, but never unconscious. Awareness is merely the more potential, more dynamic consciousness. Or the other way around: Consciousness is the more static awareness.

Stop, stop! What does subconscious mean?

That means conscious below our consciousness in the stricter sense, nothing else than dynamically existent, only conscious as a potential, as a potential to realize a potential, et cetera. Although always as the side of an alternation, or it is literally "out."

Okay, it's all potential. "I am aware of something" then means "I am aware of its potentiality"? 
Exactly! We are always talking about the same here. Just don't let confuse yourself!

Well, I'm not sure. Normally, we just don't assume that the subconscious is always accessible to us.

How then do we know it is there?

Because we conclude about it from what is happening to us.

That's it. We reasonably imagine a complex something, which exists "about there" for itself and occasionally makes itself noticed. This is potential existence, with all uncertainties such a potential brings with it. We could, of course, find straw as well, if we have a look.

\section{I understand. Is potential existence the same as dynamic existence?}

Only the emphasis is different. Dynamic existence is the generic term, but potential existence we can say if we consider more the potential as such, instead of the alternation. Dynamic existence would mean the subconscious into which we put ourselves. So, also a greater certainty would be described, but no absolute.

Well, that has always confused me a bit. Maybe this is a good crossing to why the slowing down of the alternation is only apparent ...

Let's try it. Have you already wondered how we organize the speeds of the whole alternation between edge points, edge and center, its center and sides, open and self-contained continuations, et cetera? And then the alternation with the rest of the universe?

$$
\text { Er...no. }
$$

This question has occupied me much. Within a finite reference frame it is relatively easy to solve. What is now less conscious to us can alternate faster. Gradations in consciousness therefore can be gradations in the speed of alternations. If this is indeed the case, it is almost negligible.

\section{How so?}

Please, don't get me wrong. We speak of a very basic process here, on which many less basic processes can be superimposed. Whether something is conscious or subconscious may depend on many structural differences where we do not ask about speeds. For example, even a very slow movement can lack the other side of the alternation. On the other hand, we do not come to the conclusion that there could be another side if we do not hurry ahead of the movement. Higher speed here means more consciousness.

\section{Or rather more awareness?}

More conscious awareness. However, if we do not hurry ahead, does the other side exist at all?

This is like the question whether the moon still exists if we are not looking.

It exists. Because on a deeper level we are looking again and again much faster than with the eyes. But even faster than in thought. Only then we can find its "track" seemingly subconsciously, catch sight of "the" moon spontaneously. 
So the speed defines the degree of consciousness anyway?

Ultimately, yes. Although the generality of this finding is a logical conclusion. We don't have to assign a certain speed to any detail of a complex alternation. For this the structures are too interlaced. It is sufficient if differences of the perception speed prevent the simultaneousness of two alternations.

There is no simultaneousness of anything?

How should it otherwise alternate with each other, that is enter into a relationship, be perceived? There can be "alternaneousness" at most, meaning back and forth or, for example, a non-independent "simultaneousness" like in quantum theory. We talk, by the way, about time again only as one possible standard.

I know. But I wonder how my perception of a candle can alternate slowly while the further existence of the moon behind me requires a much higher speed of alternation? Don't both alternations run at the same time?

In this case there would be no connection between them. As soon as they affect each other "something" alternates between them, and this is nothing else than a holistic perception focus. Even if it would be merely a "particle": It leaves a different totality and leads to a different one. A wholeness becomes another.

The candle becomes the moon.

In principle, yes.

Physicalists will be tearing their hair out!

They rather look after details. Even if they talk of light for which there is, by the way, no physical time; or of fields and entropy, they need to shift their perception from the candle to the moon in order to abstract a thin connection between the two. Doing so they perceive like you and me: Individual entireties in alternation.

So, there is an alternation between all alternations as well.

And since there cannot be simultaneous alternations all must be one single alternation!

But now you have a problem!

An interesting question, isn't it? How do we get together the alternation speeds in an infinite universe in such a way that they pass into each other without contraction?

\section{Enlighten me.}

I have a joker.

I knew it.

For its wholeness an infinite universe needs an infinite alternation speed. Besides, I don't believe that our concept of speed has an infinite shelf live. But we have to work with what we have and prove its 
consistency also with the help of extreme cases. And something more general than alternation we do not have.

In fact, the infinite alternation speed, which we have already introduced with the consciousness unit, provides a lot more options. A fast alternation may seem slow by repetition without reducing its speed: The entirety circumscribed by the form of the alternation changes without any hurry. Even if it would be circumscribed infinitely fast. More than this, it could also change itself infinitely fast and would come as little into conflict with its infinitely fast circumscription.

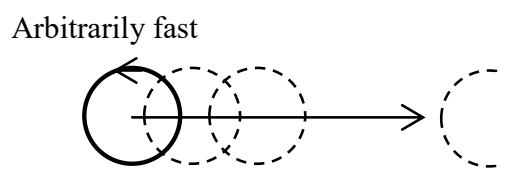

Arbitrarily fast

Infinite plus anything is infinite again. That's why I have no problem with the universe. An infinite alternation speed can circumscribe everything.

One moment. Slowly ... Complete repetition, therefore total self-containment, does not exist, you said.

I roughly said self-containment and openness are also identical. Like the sides of a consciousness unit: By immediate alternation to each other.

I remember. The circumscription therefore is as open as closed.

Yes, but the more the unity is emphasized the more slowly or statically it appears. The i-structured spiral becomes narrower, so to speak, or is just turned crosswise.

... and yet does not lose the connection to the rest of the universe.

Correct. Cross-turning stands for becoming more conscious, more quasi-static.

And now you take the infinity to unite everything that does not fit together?

Only for what can be derived from the finite and according to current understanding can seamlessly merge into the infinite. As mathematicians do.

Well, I have one more question: On the first day you said all sides are linked also immediately.

Because they as entireties need, strictly speaking, only the infinitesimal center between them for their distinction, and the same applies to any intermediate stage. This is the i-structure.

That means we have an infinitely fast alternation in everyday live as well?

That's right.

NOW I am shocked!

Come, come, we also said that alternating structures define a consciousness unit. 
In their center! Now, but it looks as if the consciousness unit is extended and corresponds to the structures themselves. A clear contradiction!

Because thus units define units?

Yes!

Okay, Okay, I admit, the consciousness unit in the center was a simplification, or rather a special case.

You don't say!

Yes, as a relief for you.

Of course.

In reality we can stick just as little to spatial thinking as to the temporal. This relief sometimes makes it even more difficult. Or what do you believe is actually an entirety? "Entire" is one, not one after the other. The entirety, of course, has a structure, but it must also be one! This only works if this identity is established immediately. So, since everything alternates, at infinite speed.

Now I'm completely confused!

We'll fix that, don't worry. The perception of the structure -as the perception of the entirety- is simply the alternation of a sole consciousness unit.

I put myself out.

Stay with me. It won't take a minute. Do you remember that an entirety can have only one central point?

Yes.

No matter how complicatedly we are alternating in detail, the whole has only one single center. How can this center probably be maintained if in between we are always somewhere else?

Okay, Okay. But the intermediate structures!

They are Inter, not the whole. Crucial for the central point is the whole! So to speak, the apex of the entire circumscription.

And?

This apex is not the center.

... But it is centered!

Precisely. A ring, for example, a ridge, a crater rim. That is what the central point most severely refers to, what determines it clearly.

Not what adjoins it ...

Not spatially, no. 


\section{But "establishing"?!}

Yes, the apex of the entire dynamic. We could almost say a psychic summit. Or an intensity peak.

It's beginning to get through me. Since a consciousness unit must be derived from a larger structure and since this derivation culminates out there instead of in the innermost, this unity of ridge and central point is the representative consciousness unit of the entirety.

Yes.

I am impressed. Accordingly, every intermediate structure which helps to build up the entirety by alternation must culminate in its own consciousness unit.

Go on.

And so the entirety is formed by one single changing consciousness unit.

Okay now?

Let's see. Maybe after the counterstrike: How can a consciousness unit create itself as an apex if it must have been created this way in order to its creation?

Now you've got me there, eh?

Well.

You are underestimating the smoothness of the infinite. The infinitely fast alternation of the consciousness unit forms -we had this already- by i-structured repetition a quasi-static focus of consciousness, which can alternate in its turn at any, even infinite speed. So, its top unit may, too, alternate at infinite speed and, in doing so, may form what it wants.

The infinite moves in the infinite ...

... and creates depending on the form of this movement seemingly slower forms.

The form of the alternation is therefore what matters.

No matter "what" is alternating here. There is, as we have said, only alternation as such.

Let's have a break.

As I see it now, every form is produced by the whole universe. Because the only alternation is moving through any form that is being created at the same moment.

Yes, and namely as an i-structure, otherwise we are silting up in contradictions. We can also say, all consciousness units transition immediately into each other, since they adjoin each other. Depending on the form of this transition, consciousness and awareness, objects and potentials originate from it.

Although we have derived the consciousness units only from such objects and potentials? 
Thus, it is. We can base our world view on nothing except on our perception. But we can explore and investigate it to make it consistent. In this case we get back complex quasi-static focuses of consciousness, which now can claim any degree of flexibility by themselves and so in turn prove to be the basis of reasoning. For only movables can enclose something.

\section{Day 4: The unlimited potential}

I have understood the origin of freedom of choice through your book "How Consciousness Creates Reality," at least intuitively. Nevertheless, it seems to me, the explanation by alternation speed is more obstructive than helpful for an intuitive understanding of i-structure. Apparently, a decision is reduced to a consciousness unit. Can this be?

... to a consciousness unit which leads to new consciousness units through the identity of both the urge to change and alternatives. It is equally true, however, that all consciousness units by infinite alternation speed establish a unity and form quasi-static focuses. Unity becomes intuitive by infinitely fast alternation, because the latter is the transition to wholeness in zero time. We may simply not forget the zero. It is not only approximated but reached. This is wholeness! The alternation is only for the connection to the difference. Wholeness and structure form a contrast which is offset by alternation.

The alternation, though, is a contrast by itself ...?

But more precise, since it includes the sides as such. As well as its own wholeness. An alternation between this wholeness and the difference of the sides is here again without intermediate stage.

So, again zero.

Not zero only! Zero is nothing without its role. The intuition has something to sense.

The alternation.

Are you kidding me?

All right. Intuition equals alternation wholeness.

Down to the infinite small, at any place. That's intuitive enough, I think. If you apply this to a more complicated entirety, you cannot longer "see" the top unit clearly, but sense it at most. Probably rather a cluster of units around.

A condensate?

Thus, we feel it. But now around the crater ring, inside and out.

Must not the condensate be in the middle? 
We consider here the apex of the dynamic form. If the condensate is in the middle, then the apex is there.

Okay, slowly everything fits together. Now I also understand better why you like to draw the consciousness funnel or reality funnel with an "outside area" like a crater:

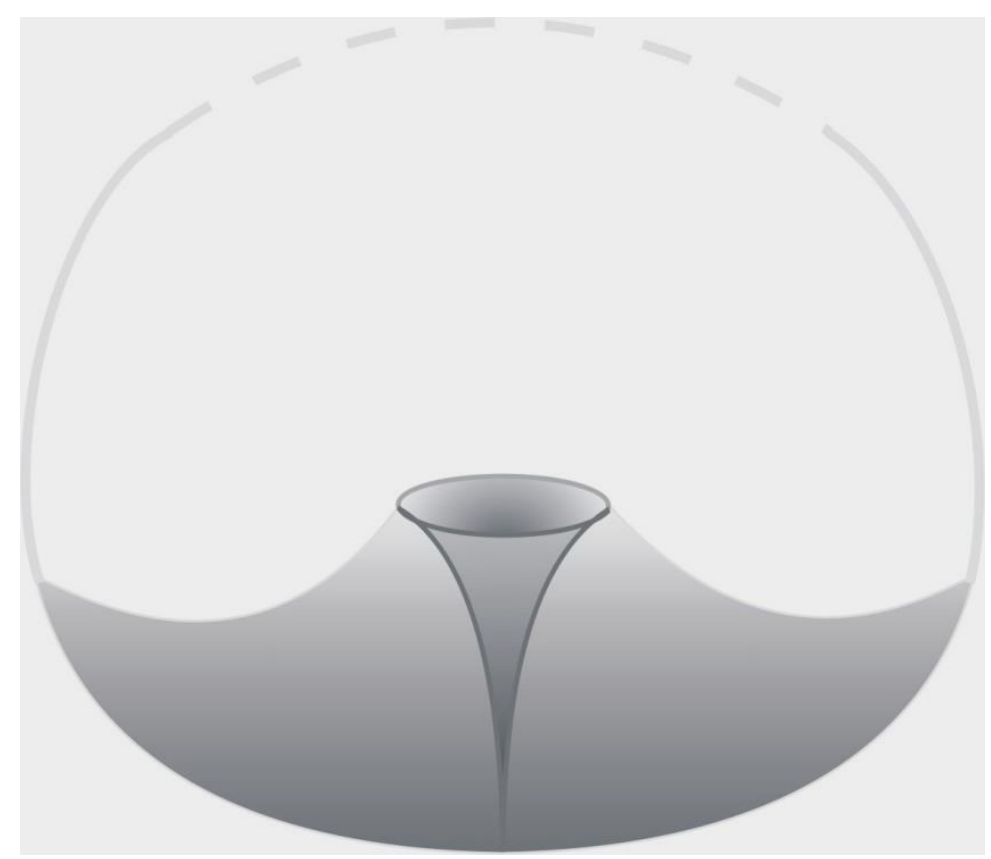

It's not really about inside and outside, right?

No, these are limited concepts. It's rather about up and down meaning more conscious or less conscious. The exact center is an axis passing through everything.

So, all standpoints or perspectives, which I am less conscious of, are located in the stem of the funnel?

The less conscious the awareness the deeper they are circling. The details are becoming increasingly subconscious: 


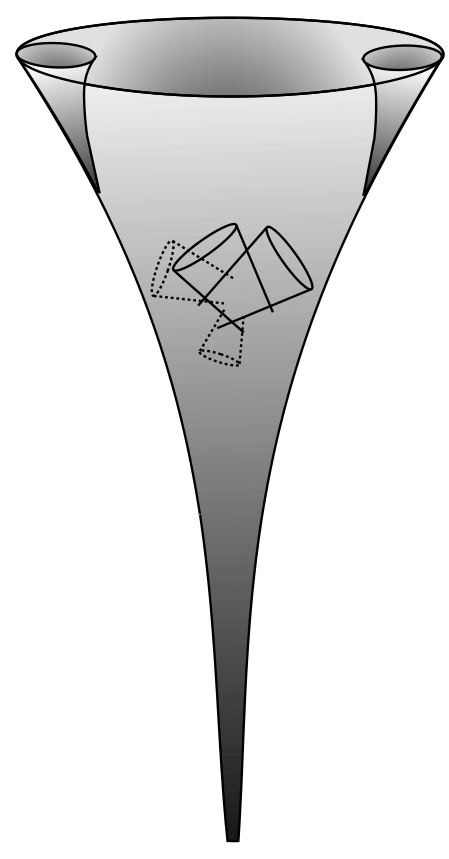

If I have understood you properly, I am not only aware of other consciousness, but of other awareness. Because the infinitely many other standpoints from which my awareness is being dynamically built are also just summits of infinite dynamics. Aren't these infinities a bit too much?

Which ones are too much for you?

I mean, how can my awareness apprehend an infinity of infinities and yet remain structured?

Because resolution at infinity is coming too early for you?

Yes.

Don't worry, it's not coming. It's already here.

Sorry?

Awareness is only structured after all because it is suppressing most other standpoints repeatedly into the increasingly less conscious. Until they almost merge with the central axis. With this axis we anticipate the infinite. It is not "counted."

??

You feared intuition coming off worst, right? Well, awareness is becoming more and more intuitive downwards since consciousness of details is strongly decreasing. The "tracks" of the alternation to the Other are becoming denser and denser and only resolvable if consciousness follows them. That means where it is at the moment they are hardly conscious anymore. Awareness can consciously anticipate the infinity only as such - in the intuitive knowledge that it is there. As the said axis or as a central point.

Or as a potential. 
Yes. Since consciousness is slow, we can consider the central point also as an approximation of the infinite, as a symbol of something to which we can "go" if we "quicken our pace" strongly, asymptotically up to infinitely.

Fascinating. Must there not be, however, "infinite space," an unfolded infinity that we can anticipate?

Of course. But it is in the opposite direction.

In the direction of consciousness ...

... and with it of the absolute universal continuum which I have explained in "How Consciousness Creates Reality."

But an absolute continuum is structureless and cannot be conscious!

So as the zero?

Hmm.

The total unfoldment of absolutely everything to a distinctionless continuum is its collapse at the same time. But to what? To a Nothing? Then unfoldment had not happened at all. The universal continuum rather "reflects" on from which it was reached: It exists only for the awareness by which it is anticipated.

So it does not exist for itself?

Only as a momentary extreme case within an alternation of perspective, like everything else. We already had this.

The continuum has a perspective?

Only in the alternation with another awareness.

Well, in "How Consciousness Creates Reality" you also describe All That Is, the highest possible consciousness. It should be located just below the universal continuum, "at the brink of collapsing." What is it doing there?

I guess it's playing God. We are dealing with an infinitely complex and infinitely large i-structure forming itself, like everything else, at infinite speed.

It is in a focus?

Not like us. Its alternation has to include everything equally. Nothing may sink into a funnel stem. Therefore, All That Is is in any focus and differs from it only by one single criterion, which solely is its own: The unlimited potential to take up a different focus. With that it is but always a certain perspective of this potential.

I have to digest this for the moment. I am All That Is?

Can you take up any focus? Let's say an infinitely complex and infinitely large one? 


\section{No. But why not after all?}

Because the shape of the alternation of your focus has become independent. It does not only seem slow as a whole, but it has suppressed and forgotten the ability to accelerate sharply.

\section{What did I do to deserve this?}

It was -like everything else- a decision. A lot of decisions, actually. All of them concern the form of focus shaping but some also concern the form of form shaping. There originated not only consciousness but self-consciousness. An ego, if you like.

\section{And the ego prevents that I take up a different focus?}

The self-consciousness creates stability by rather choking the awareness of the greater potential and letting pass merely vague ideas. But you may certainly put yourself into the position of a coffee making questioner and bring me one.

Sorry, I'm on my way.

Thank you. If you have placed your focus then again, please tell me, why didn't you go earlier?

\section{Hmm ... You mean because I am selfish?}

Just a bit, of course. You were absorbed and I appreciate that, because it had a meaning: You wanted to grasp, concentrate, and be wrapped up in your part. This is why we do something like that: We create structures which do not collapse immediately. The whole universe does it. Otherwise, it would have remained in the continuum.

\section{Where it had gotten to, even though from a structure?}

The classical alternation.

Where had we stopped?

You had choked your awareness.

\section{Ah, how did I do this?}

By reflecting on yourself again and again from birth on at the latest. What I find Okay, by the way. By discovering ourselves anew we contribute to the awareness of All That Is as well.

However, All That Is is not in my ego-focus, as this one has merely a limited potential. So what rewards does All That Is have?

That's just the point: The infinite focus speed also encompasses any self-consciousness. By the latter the seemingly slow focus has, indeed, clamped itself to a great extent. But since it moves on, changes and develops, it reaches its infinite potential again in the infinite at the latest. Yet, because we are aware of this infinite as a potential to a potential even now -of All That Is as such- the self-conscious focus alternation as well must be a sub-frequency of the all-encompassing dynamic.

Would you like some more coffee? 
I'm in progress. Now the million dollar question: How can that be?

How can what be?

I'll tell you: This is nothing but the typical dynamic existence!

$A h$.

How is it characterized? The other side of the alternation we are always only aware of. This exactly is alternation: Everything in the either/or.

What means ...

... All That Is can always only be aware of our focus.

In order to do this, must it not really alternate with it?

Certainly, and only to us this alternation is conscious so little. So there must be still another way of alternation, which we are using even less consciously.

Well, the consciousness units have, indeed, found one which the slow focus hardly grasps.

You always surprise me. So: Between infinitely fast consciousness units and self-consciously bound focus there must be at least one other focus alternation with the infinite, which escapes us according to form and speed of our own focus and, for example, provides for the feeling of a "divine presence." Such focuses are constantly removing from us and passing into us again, without that we are seeing ourselves in the situation to "follow" them.

That's strange. Since now we are not dealing anymore with infinite speed, in which everything can level out. These intermediate speeds are finite! Do they not get in a mess?

Why should they? Our quasi-static alternation, our most superficial focus of consciousness, is not completely isolated, as you know. No matter how self-reflexively interlaced it is. It still forms out of the infinitely fast alternation of consciousness units. The accesses to other forms and frequencies merely escape from it. It skips phases of the whole alternation, as we forget our dreams. Although it has basically permanent access, even to All That Is.

We just have to find it ... And be able to cope!

Yes. We can cope with it only well measured. Otherwise, we lose ourselves this time on the other side.

They say we are protected.

This would make sense. Even All That Is needs relatively stable structures to alternate to and to be aware of. It is diversity, not chaos.

I assume, by All That Is not only the consciousness units are meant, but also the focuses infinitely fast for their part? 
Yes, all of them; and the slow! Who is on the move fast, can be slow within that also, by temporary repetition, as usual.

Focuses in focuses in any repetition?

It doesn't matter. This makes a difference only when we become finite. And then we still have an infinite span on which the speeds can spread. Since All That Is does not become finite for sure.

And so we are only apparently finite.

But this appearance is very real since it is necessary even for All That Is, do you understand?

Because it would, otherwise, evaporate in a continuum?

Got it. So, "bogus" can actually be no question. We are certain structured phases of the overall movement of the highest consciousness, individual awareness which All That Is, too, is aware of, but in its individual manner.

All That Is an individual?

Of course. Who else is characterized by infinite potential?

The universal continuum?

Good thought. Both universal continuum and All That Is need us for their determination. Yet, the continuum does not have its own existence. All That Is does. It has awareness and condenses for us barely to a consciousness. It forms the state of reflection of the universal continuum. It is the big brother of the consciousness unit at the other "end."

You say "It condenses for us." Doesn't this show its dependence?

Without us it is nothing! But we and all other focuses of consciousness create it as a structure, as that what creates us.

\section{Is this fantasy then?}

Not more than the perception of our own existence.

I understand. It is on the consistency of the perception.

On every conceivable level.

Still: Has All That Is an own consciousness or not?

Since its awareness apprehends every other at infinite speed, it couldn't be more conscious! Nevertheless, most of it is always just subconscious, for it remains individual, as you know. Even for All That Is! But it can condense only for us, from a restricted viewpoint.

So, if All That Is is in a certain focus, it is condensing for itself?

If it is not using its potential, it is just not All That Is anymore. It is only a focus with a condensed potential for higher things, briefly: With a condensed imagination of the highest consciousness. Even if this potential is available at any time. But if All That Is is using its potential, it is this potential. 


\section{That's heavy stuff.}

One thing with this whole focus alternation model bothers me, though: In order to explain relatively small processes we have to deal very quickly with high alternation speeds. In my mind, this challenges the plausibility of the concept.

That's another story. In the beginning, new theories seldom give rise to good feelings because they are simply unusual. This one is consistent, as far as I can tell after years of investigation. Whether it is applicable to all putatively material things in detail we should explore motivated by this logical consistency.

Please, also keep in mind how fast we can change whole scenes, for example, in a dream. And these are rather snapshots. We may not stick to a movement idea that arose from the carriage age. Even the speed of light cannot be a serious barrier outside the well-known space-time, if it ever was. We don't send information, but alternate entireties.

Into the unknown, but you can project anything.

The only question is whether it harmonizes with known processes. Infinitely large things we just don't need in practical terms. We are talking here about that extensively only because we, as already said, test the consistency with the help of the extremes. On the other hand, we do not make the whole stranger than it is, anyway. Normally, we just accept it.

Yes, we accept a lot and become uncomfortable if someone questions us.

I would like to prevent a misunderstanding, though: Wholeness remains intuitive in my model as well! Because the described transition to wholeness is not its derivation, but the connection to it. Wholeness and structure are not derived from each other but are sides of alternation. Just as the central and turning points that we have already discussed. Consequently, without intuition there is no alternation.

And no structure.

Nothing at all.

\section{Day 5: Indestructible}

I had to think about what you mean by culmination, apex, or summit. How can the starting point of a perspective, an intensity peak of perception, arise from alternations? Mustn't I assume a certain peak to become aware of an alternation?

That's how it is ultimately. We go out from a perception. It is holistic and therefore unique, because any comparison with other wholes would create a new whole. Hence, this unique wholeness of our perception is always the starting point. It is the current intensity peak. Everything we then investigate 
are relations with other wholes, whether partial entireties or being outside ones. Just the alternation of wholes.

\section{Couldn't there be a higher partial summit within our wholeness?}

We can define the summit this way: It is the conscious aspect most strongly determining the awareness. If it is conscious less, it cannot be the apex of the awareness, because we are always talking of the conscious. On the other hand, if it determines the awareness less, it is not the most intensive unit.

But it is also the result of alternating consciousness units, focusses, and so on?

Yes. Only the starting point is not. We go out from the summit and examine its relations with other summits, which lead back to our summit. However, by doing so we have extended our awareness and thus have created a new summit. This is the real result of the movement. Reaching the starting point would be more difficult, though not impossible.

We are yet again with the i-structured spiral.

We cannot escape from this. But now we see better that the summit is not the largest consciousness. And how should it be? It is the funnel edge, not the funnel. It could even lie closely around the center. Instead, it is important that the relevant decisions emanate from it.

Ah, I understand. That's why it has to be the most conscious aspect: Because decisions are made consciously by definition.

Or because everything is consciousness and therefore decides constantly.

So, if a partial summit would be higher, more determining, the larger whole would be less conscious.

Yes. Although it's not a partial summit then, but the overall one.

Okay. Another question: We found that the movement of an entirety follows the decisions of its $i$ structure. It has very different alternatives: Slow and fast; this or another direction. How does it happen that this movement "skips" a faster alternation in favor of a slower one, that the accesses to higher frequencies "escape" from it, as you said?

As you know, alternation happens in leaps and bounds by nature. Apparent continuity comes about only by close together experienced entireties. Therefore, we can also make immense jumps. Let's say, from the couch to the star system Alpha Centauri and back to our juice - while only the couch and the juice are related to each other, and everything else is taken for nonsense. Thus, it is the assessment, the selection of the summit from the overall alternation, that makes the difference.

And who makes this choice?

We do this by deciding on priorities, on a certain perspective of the overall alternation. In this perspective we are aware of the stars only distantly. Instead, we have turned the alternation between couch and juice crosswise, slowed it down, and made it quasi-statically conscious. 


\section{Did we change the direction of the overall alternation for it?}

We have designed a certain alternation out of other alternations. Thereby, we have restructured the overall alternation. Yes, yes, to infinity.

We always have a perspective ...

... and we change nothing but this perspective. From the infinitely large and fast we could in principle design everything - by decisions of the already designed - if we would not have interlaced ourselves to an extent where we have little idea. By choosing a new form of the alternation we choose a new wholeness and a new perspective, and vice versa:

$$
\text { Form }=\text { Wholeness }=\text { Perspective }
$$

A chaos or continuum cannot choose anything. So, the choice must ultimately originate from All That Is as the largest form, the largest consciousness at all.

Precisely. In the end, everything is a "divine" choice, but not without asking us.

\section{All That Is creates us to ask for our opinion?}

Not only for our opinion, but for our decision. Everything is i-structured and hence decides constantly between alternative sequels of its alternation. Intuitively, holistic, mind you.

Whatever that means in the case of my saucer.

Your saucer has been created to stop your spilt, not to make its own way. It may decide at best within $1 \mathrm{~mm}$ play left or right of the cup.

\section{But how does it do this? It is made of porcelain!}

How then do you come to think of it being a saucer?

I perceive it as such.

Only because of that it is used as such, only because of that it "is" such a thing. So if it wants to move to the kitchen, you do with it.

Because it exists only in my perception as a saucer ...

Exactly. The decision to switch over to the kitchen is made by the entirety of the saucer and the coffee drinker and other circumstances. Here, you undoubtedly have the privilege to be allowed to identify with the summit of the situation alternation entirety. So, with the most determining aspect. However, if the coffee turns out awful this may change temporarily.

Will the coffee then decide that I will pour it away?

In the entirety of coffee and drinker the coffee has temporarily taken over the direction. You, by contrast, have decreased to your reflexes. Only afterwards you remember your veto power and perhaps still drink it. 
What if I do not touch the coffee at all? Does it decide to evaporate, or does it do this simply because it is hot?

I would say because it is hot.

So, no decision?

I am not to judge whether it cannot decide on one or the other damp streak. Just as little in which manner the saucer is involved in whether it is set down $1 \mathrm{~mm}$ to the right or the left. Also, physicists can't do that honestly but refer to the chaos theory.

... which makes infinitely small triggers responsible for bigger changes.

But if you restrict a situation until only minimal margins remain you don't need to wonder if this is the case then.

That's interesting. Since, actually, we see it just the other way around: The world is relatively rigid, and we are flexible and conscious enough to make active decisions, freely or not.

If they are not free they are no decisions, no choices, by the way. Why your coffee evaporates at all I can explain only physically, too. But now I can ask why such a thing happens under similar conditions always similarly if it must be a chosen event by general logical considerations. A physicist, by contrast, would stop with the statement of a so-called physical law.

So, the question is: Who has chosen this law and why? Or should we say "enacted"?

Since the answer is not conscious to us we must look into the subconscious. By that I mean, of course, everything conscious, which we are aware of, but as good as not consciously. Everything in the stem of the reality funnel.

Up to All That Is?

Yes, because, as you have already stated, everything we perceive has its origin there, respectively is connected to it. By the way, I think "enacted" makes more sense with the stability chosen at the same moment. Hence, we have neither to think with every step whether the ground holds nor whether the coffee freezes with the next gulp. That's reasonable, right?

Quite.

So, if we can agree on the fact that everything is basically chosen, the question why there are regularities - another such concept delicacy - can surprise us just as little as a physicist. We must look, like him, only after the details. Although with a different emphasis.

A spiritual emphasis.

And without excluding physics. It is the result of unknown decision-making processes.

However, as for the spiritual details we are groping about in the dark.

Because we are not looking for them. If we would make the same research effort in this direction as in physics, we would be further ahead in all respects. 
Okay. Can you briefly summarize this decision-making process starting from All That Is?

Well, All That Is, as the most complex possible structure, has not only the largest selection of alternatives but also the highest density of conscious moments of choice. It can choose anything at all, it is pure potential.

Is this the same like energy?

No, energy is abstract. Potential is always on concrete, at least in this context. Even if all concrete is meant it is not assimilated to a flow or congestion, but remains in the alternation as such.

Agree. So, what does All That Is choose now?

Since it has to unite unity and structure this altogether amounts to a hierarchical order. Other variants may not claim the equal range of existence. They don't have the same degree of order and many coincide qualitatively: A fog bank hardly differs from the others and can claim in the spectrum of possible orders not as much space as more structured variants.

This is pretty much the opposite of what most physicists say.

They think differently. They don't really combine microstates, but count their combinations. Thus, they conclude a high probability of chaotic states. And then they need a not really explained "big bang" to put down the presence of order to something at all. But where does the order of the big bang come from?

It resembles a bit what you have just described: Order because in the end nothing else is left.

A little bit, yes. But it is a dead and limited order that lacks the consequence, as we have seen. It lacks wholeness, alternation, consciousness, openness ...

... and thus freedom of choice.

Although it is, of course, a choice of each researcher to pay this price and to abstract from the individuality of perception and his own consciousness.

\section{Let's continue with the freedom of choice: Does order still arise from it?}

Where would we end up, otherwise? Seriously: We could hardly grasp comparatively large agglomerations of consciousness, which is why we are aware of them only far below in the stem of the reality funnel, as a deep idea, so to speak.

However, this idea could be very vague.

I would call it a more infinitesimal i-structure. This is different from a condensate. The alternation paths with the deeper are maintained and are just closely bundled. Thus, we can find everything again if we open ourselves. But only with a certain probability.

How is that?

Alternation $=$ consciousness $=$ freedom of choice . Do you remember $?$ 
So, we don't know if we choose the right thing when diving?

We don't know if our subconsciousness will choose the right thing. Or if what we are looking for has slipped away in the meantime. At least, we may consider the next large consciousness agglomeration in our subconsciousness as an entity. We arise from it in the funnel image and alternate constantly into it and back, but we hardly remember that. It's rather like a dream-like sensation, or intuition. I have described this in my book How Consciousness Creates Reality. The Full Version in detail.

Yes, the entity is for various reasons more stable and therefore our anchor base.

Since this is more conscious to the entity and we are its "children," and since it holds the role of the "guardian of order" in the hierarchy of our entireties, it will determine or inspire our behavior to a considerable degree. It provides impulses, which reach us out of the funnel stem, and to be seen "from above" they are difficult to separate from our own choices.

Only if we exert our self-consciousness some things become clearer.

To a certain degree. But in the end impulses and choices merge.

So, we may not hope for an ultimate decision between determination and freedom?

Ultimately everything is free - chosen by All That Is. Although by us, as we know us, only in a relatively small part. And we often don't even know in which one.

This doesn't sound like conscious reality creation.

Compared to a through and through determined or random world it is much more what we can create! However, the decisions of all consciousness slip in and prestructure the probabilities available to us. So we operate in a hierarchy of probabilities, which we restructure by our decisions, as all other individuals do.

That's why we don't fly to Alpha Centauri, but stick to our juice on the couch.

Obviously, a high probability has arisen from the whole tangled awareness for the alternatives water or juice.

When you say "create," do you mean only a free choice?

Basically yes, because this is what entails everything else. But creation means figuratively also emphasize, lift out.

What do we lift out from what?

We lift out the priorities, the respective intensities of our life from all others by restructuring our hierarchy of probabilities, our perspective, our awareness, with every movement. Since everything is linked, this change reaches into the infinite. We create, so to speak, a new universe at every moment.

If we create a new universe, what was there before?

All other perspectives, of course. They are still there as well. There are, as you know, only perspectives of the overall alternation. The term "universe" is misleading, because it suggests one 
same world for all. Such a thing can't exist. Also, nobody lives in an own world bubble, but in an individual awareness of the overall alternation.

I must cut in again.

Sure.

But a hierarchy of priorities is also a hierarchy of different alternation speeds. How can my awareness, so my very individual hierarchy of alternation speeds, be a part of your individual awareness? Obviously, you can't experience my focus with the same slowness as I do, but only your own.

Ingenious objection. But it depends only on the individual differences of the alternation speeds. So, I am aware of your focus really faster - and less consciously - than you, but basically nothing prevents me from being completely aware of your hierarchy of speeds. Because, although they are shifted for me "backwards" your speed differences are preserved to me to the infinite. Infinite plus X minus Y is still infinite. The fact that to me your awareness is less conscious is self-evident. We have spoken of this.

That is ... The infinite would thus preserve the form of my individual hierarchy?

Of course. All That Is isn't called so in vain.

But the universal continuum ...?

... is only a single standpoint in the alternation of All That Is, furthermore, one which has meaning only as an extreme value within this alternation. I cannot rule out, though, that it reflects on something else than you, but you are preserved, since All That Is alternates through all possible hierarchies. It is unlimited potential.

So, there is no destruction, not even by resolution at infinity?

No, there isn't. What we have carelessly called "resolution" is a passing disappearance in the infinite "distant", to the consciousness in the infinitely little accessible. This "point" of disappearance can be also an infinite alternation speed, which we perceive as an infinitesimal wholeness. The infinite fulfills both functions: Reconciliation and recoverability of the opposites.

\section{Day 6: All-Encompassing creativity}

I have noted a brief summary about entirety:

Every perception is individual. On the one hand, because it is only possible from a single perspective, and on the other hand, because it intuitively unites the perceived structure. If we change the perspective we therefore change the entire perception. To put different perceptions in relation to each other we must alternate between them and form thus a new intuitive entirety which contains the 
alternation as such. However, this was already valid for the first perception, which is why it is always about the entirety of alternations.

Fortunately, we perceive this entirety largely diffusely as a condensed unity, particularly if we look at the alternation crosswise, but even if we are in its line. In the latter case, the condensate camouflages our potential to reach the other side of the alternation.

I was not aware of having expressed myself so clearly ...

... But if we want to be precise, we must follow the alternation to the infinitely small to analyze its entirety. In doing it so we find that different levels of structure are formed at seemingly different speeds of alternation up to the infinite speed of the consciousness units, one of which is also the summit of our seemingly slow perception. With it the last connection between the intuitive entirety and the sides of the alternation is established.

Amen. I congratulate you! What shall we do with the rest of the day?

I'm still left with a few questions.

Go ahead.

You said All That Is has a structure. If it alternates through everything and is thereby pure potential, how can it have then a certain structure?

At the risk that you suspect me again to abuse the infinite as a projection screen: Yes, we can indicate main features of a probable overall order resulting from its combination ability and of its development results. This is the hierarchical order of entireties intimated yesterday, the production of limited offshoots of the awareness of a more comprehensive entity. All the other variations of alternation remain untouched; we will only deal less with them.

So, we don't prescribe All That Is how it has to alternate?

No, all alternation paths are equal. But we see this one more often than other ones because this creates less inner contradictions.

And this does not restrict the potential of All That Is? And thus, freedom of choice generally?

No. One cannot demand, though, that self-created contradictions are cured at the same place. If I want to go to the left and right at once, this is a contradiction in itself "within the framework of my existence." I can only resolve it by extending this framework, so for example, by soaring up or by splitting up myself. If I expect All That Is to prefer alternation structures with little association to me, it will just not do that. Since for me only exists what in the broadest sense fits me. We create God in our image.

It seems to me that you have missed your profession.

You're telling me!

Nevertheless, as a result we have now but a restriction of All That Is. 
Only for us. We have tried to see something concrete in All That Is. This is justified because we have a relationship with it. But it is already a condensation, even if for us it's the most general one.

Our extraterrestrial friends could therefore recognize other most general structures in it?

To do this, they would probably have to be so alien to us that we can hardly speak of friends. But it is not ruled out.

And a much higher developed entity could have both versions assimilated into an even more general one?

Yes.

Then All That Is could be also something completely different from what we believe?

Absolutely. Here we go with what we know as far as possible, not more.

Okay. So, the reality funnel is the image of our narrowing knowledge, isn't it?

Yes, with the narrowest point standing also for the universal continuum, which does not know differences.

Hence, it is completely "inside" as well as completely "outside."

Yes, and only an infinitely little step -an infinitesimal "alternation step"- closer to it we find All That Is. Abstractly, we may imagine it as an infinite large complex. Nevertheless, its concrete complexity is in the funnel stem, in the subconscious, where we are aware of it as compressed almost to amorphousness.

Isn't this more an overlapping of details in perspective?

Yes and no. We can shift arbitrarily many details "backwards" since we speak of the smooth infinite. However, if we disregard this -to be fair because it already concerns the most complex structure- we still compress it to a diffusely appearing potential. The pure dynamic of All That Is unites all structures as such at infinite alternation speed. For us, slow cosmopolites, this speed appears as an extreme density.

It appears so to me as well.

At the end, "compression" is a metaphor that includes much of what we encounter in this context: Entanglement of alternating perspectives, enfoldment of structures, and condensation of dynamic. Or in the reverse direction: Expression in slower forms, expansion of consciousness, and disentanglement of awareness. - Well, whatever: The dynamic of All That Is is what influences us the most in the broadest sense.

And yet, All That Is can be topped by our own unity with the universal continuum, if I have properly understood you in "How Consciousness Creates Reality. The Full Version." Because out of the unity with the center, we decide.

Is this a question? 


\section{Quite a statement. Or do you disagree?}

No, it sounds a bit mystic, but it's true: In the universal continuum and thus in this deepest certain point of the funnel everything merges as well as all other funnels. This point exists for every awareness. It collapses with the center of every alternation from which i-structured unity we choose. We perform therefore basically the same act of choice as All That Is, and so we can even oppose "God."

Considered conversely, though, countless "divine" choices also manifest themselves in us ...

... which is why for our consciousness relatively little is left to be done. If we consider, however, our entire focus hierarchy as what makes us up, "we" decide about literally everything that happens to us.

That sounds more like reality creation. What about the decisions of the other funnels?

Since all other awareness also crowds in our funnel stem, all alternatives and choices of these individuals do it as well.

By "individual" do you mean the entire focus hierarchy of an awareness?

Simply awareness. It is, as you know, always a unique, indivisible perspective of all.

$$
\begin{gathered}
\text { Awareness }=\text { Reality Funnel }=\text { Focus Hierarchy } \\
=\text { Consciousness Hierarchy }=\text { Probability Hierarchy }
\end{gathered}
$$

Have I missed out one?

I know, you only describe different aspects of the universal alternation. As for the hierarchy of probabilities: It contains also all the individual worlds between which other individuals decide, just as those which we have not chosen?

Since there can't be final limits our awareness must contain simply everything, just All That Is. By far, the most of everything, however, it's not conscious to us - at best it's a residual feeling and in any case is not as the other individuals are aware of it. Everything is re-mixed if we lift out our perspective. On the other hand, we have only alternation and arise only from it. We remain thus aware of the individuality of the others. The transition to them is i-structured; so please don't fix me to one side.

There was still something with the i-structure ...

Right; such a deeply sensed idea I have called "more infinitesimal i-structure." There is not another word for it since this concept is fundamentally new. It is not about a condensate, but rather a "sensing of the path." Of course, we always condense something, but this is not the point here. Instead, it overlays it. The deeper awareness is camouflaged by the approximations which we form from the more superficial part of the alternation. Casually speaking, cars camouflage climate change.

But a few days ago we have clarified that we can anticipate the infinite only as such. Yet, the individuality of most other worlds gets lost for us? 
I can only appeal to your intuition. We anticipate the transition to the infinite, meaning this act, not only the universal continuum or the central axis. Alone they would have no meaning as we have already noted. In the transition nothing gets lost, though. I-Structure is just this total unity of definiteness and indefiniteness within every not total unity. So, we can continue this act of transition on and on realizing infinitely much.

Although we do not know what it will be? On the one hand, everything is already there, and on the other, it is not there?

Everything is in All That Is. However, All That Is as well can be merely aware of the most, since it remains individual. We have said All That Is forms the state of reflection of the universal continuum. It $i s$ this total unity of definiteness and indefiniteness and less total unities. It is choosing and forming.

So, all infinite awareness is contained in All That Is, even if it is infinitely remote and subconscious. So, even if every choice is free, it only creates what is already present somewhere in the allencompassing alternation.

Yes. Is choosing another infinite universe not creative enough to you?

I think neither of us is too modest. Isn't there a bit more?

$W e$ are there.

I see.

Really?

Maybe you could explain it anyway?

Yes, we slowpokes afford what is not possible for All That Is: To go a way which is freely enough chosen as well as unique. All That Is must become us in order to experience the way. It cannot take this way because we are choosing it.

Are we not even a part of All That Is?

We are, but in a way that ensures our all-encompassing creativity - and I think, also of those of all other individuals:

$$
\begin{aligned}
& \text { Creativity }=\text { Slowness }+ \text { Freedom of Choice } \\
& + \text { Infinity of Awareness } \bullet \text { Infinity of the Way }
\end{aligned}
$$

If one of the components is missing, the way of the individual either was already gone or is repeatable and without the infinities even "below" All That Is.

Why can't it be repeated?

Because then it must already be known. But on the one hand, nobody except All That Is can take up and hold with certainty our entire hierarchy which is going this way. And on the other hand, nobody not even ourselves- will eternally make the same decisions again. Not even for very long, since for 
doing this the conscious awareness of the further way had to expand exponentially - due to the many possible divergences. The relative slowness of the movement would be jeopardized soon.

Or we do without a large part of our freedom and let us guide ourselves by All That Is ...

This, too, would be a permanent choice. However, a minimal residual probability of special cases is not sufficient to question the creativity of almost all slow ways.

And All That Is in turn is too fast for these ways.

\section{Day 7: Free will}

I would like to revert to the freedom of choice. I wonder how conflicting decisions of two individuals can lead to a free choice of each. For example, if I decide to continue this conversation and you decide to end it, are we both still free?

A contradiction arises of course, in so far as it is harder for me now to charm you out of the room. And it is harder for you now to persuade me to go on talking. Certainly, we could refer to the question why we have brought ourselves in this situation at all. A basic solution, however, one should not shift "backwards." It must also apply here and now.

That's what I was getting at.

So, apart from the origin of the situation, in it we -as we know ourselves- must still have a certain degree of freedom.

Not only in it, but everywhere! Because such conflicts should exist on all levels of consciousness. In any case, on the finite ones.

Okay, but one after the other. And let us better speak of awareness, because this refers directly to the transitions between all individuals.

Agree.

If my decision requires a quite specific decision of you, this world in which you decide exactly so and not otherwise must be realized in an ultimately limitless All That Is. And the world in which you decide otherwise must be realized, too, because I also could have decided otherwise - in your sense.

So each version is realized. Just as in the many-worlds-interpretation of quantum theory?

The quantum theory in its today's form offers at best similes for these events in everyday life. We do not need it to explain them. We know anyhow that every world is individual and must be realized if the universe has no final boundaries. With the i-structure we also have a reason for the proximity of the alternatives, and instead of chance we have a real choice between them.

But do we split up our world as well as in the quantum interpretation? 
"Split up" doesn't hit. Since for the so called past basically the same must apply as for the present. We speak of different development or decision-making processes meeting and separating, and between which a constant alternation is taking place. The perception of all that is the individual awareness.

So am I, though I am staying here, also aware of the world in which I am gone?

Only less conscious, yes. I am, too, aware of such a world, but in my way. Just as in a world in which you are gone this continuation of our talk is less conscious to me, rather dream-like, as a vanishing potential.

Well, but you could completely and utterly decide to let me throw out. And I could do nothing against it in the end. Where is my freedom here?

You could do very little against it. Nothing is complete and utter here, otherwise you were already off. Yet, the question is legitimate. Remember All That Is taking up another infinite awareness at any moment. Such an awareness corresponds to your reality as well as to mine and to our collective approximation in which you are here as well as to that one in which you are off. There are ultimately no contradictory realities, because they are not forced to unite under contradictory conditions.

By "contradictory" do you mean again self-created "contradictions in themselves," not colliding decisions?

Yes, contradictions to the own preconditions; such as when we both would insist on prevailing in the same reality. Then it ends with fisticuffs and at the latest, when the police arrives, it is done for your freedom.

There are yet no same realities...

No, that's right. Only if we start to fight we mutually restrict ourselves because this was not really what we wanted. We both have tried to limit the freedom of choice of the other. This is the contradiction in itself here: We cannot want conflicting things without having to leave the other an alternative. This is a self-constructed problem.

According to your theory, this problem should dissolve, nevertheless, by one ultimately agreeing to lose.

Only just too late to prevent the unwanted situation. If we allow getting to the extreme, we both can lose by killing each other. But we both cannot win without changing the self-chosen preconditions.

Then the question is: How do we attune to each other to let arise a certain situation, to maintain it, and to change it?

That is, to form a "collective" reality, an approximation -individual in its turn- of the alternation between individuals? How do we come to agree on anything? How do we make a common choice? 
Of course, we could say again: All That Is decides as a whole and we are the current summit which is involved in it and experiences the result. Yet, we experience ourselves as different individuals and want to know how these single phases of the all-encompassing alternation co-operate.

Exactly! Let's have it!

I take back the term "focus" because it is so nicely simple. Actually it is, as said, always about awareness, its alternating conscious summit.

Focus is fine. A movable consciousness within a more diffuse totality, I can imagine better.

The focus alternates between partial aspects of a more encompassing focus as well as between this whole and the parts. It is one single alternation.

That describes the overall situation?

Yes. For example, we alternate viewpoints if we imagine alternative continuations, if we put ourselves a bit into each other's position, if we are discussing. This dynamic situation has an overall central point and every partial focus has its own. And everything is i-structured.

Here we go!

Out of the unity with these centers, decisions are constantly made. However, since the partial focusses are integrated into the definition of the overall focus, their relation to the overall center point is greater than to any mere point of contact between them. Correspondingly, the partial decisions are more strongly linked to the collective decision.

What does it mean "linked"?

We speak of i-structure. Unities of circumscriptions and central points are formed, which are always total at some place, meaning in the partial circumscriptions as well as in the overall circumscription and in every intermediate circumscription. And since the circumscriptions become denser towards the collective center -their relation to each other increases- the decisions are more strongly identified with each other there.

Is there no other term?

No, it's the same as for "i-structure". The i-structure becomes denser. With it everything is said, actually, since by this way the partial decisions become one single major decision.

\section{Without separation?}

Separation is caused by suppression into the subconscious. But we speak of conscious decisions here. These originate from the unity with identity, from the identity of identity and distinction.

So, do all decide in one single act together?

Thoroughly made conscious - yes. Only the constant suppression into the less conscious gives the impression of different decisions. And now don't give me the alternation speeds! How they fit, we already have sufficiently discussed. 
I don't. But you must admit that the biggest "part" of this decision is again made in the subconscious.

It is ultimately the decision of All That Is, even to which a lot is always subconscious. How should it be different for us?

Fine, I'll try to summarize: A decision is made out of the ultimately total unity of the alternatives individuals, individual paths, et cetera-with their common central point and with the pressure from the continuing alternation.

So, we have discussed it a few days ago.

This constellation also applies to every partial aspect of the overall alternation; but because the parts are related to their entirety the said constellation is more concentrated around the overall center than if it were merely a loose aggregation.

The convolution of the whole concentrates and thus that of the overall decision to be made. But you may calmly call it i-structure.

Is the i-structure being decision in itself, so to speak, thus pulled together making its choice as a whole?

Exactly. It is useless, of course, to locate the center somewhere in the room between us. It is just not about spatial relations. In fact, any extent is merely a metaphor for the traceability of the alternations. We have infinitesimal centers at any place, even "between" part and whole, whatever this might mean "spatially." If the i-structure becomes denser, the unity of identity and distinction increases, which reduces determination and randomness, and raises the freedom of choice. With information transfer and similar movements we cannot even roughly explain this. But they are partial aspects of this event.

Do you postulate therefore a whole new process beyond transmission and simultaneity?

Not beyond, but including them as limited perceptions, abstractions, approximations. What would it mean, for example, to make a non-violent joint decision even though we are initially at odds? We would talk to each other, exchange sensitivities and arguments, that is, so-called information. In fact, individual standpoints are alternated, summits of awareness. We change our potentials and their meaning for our potential and so on, feedback loops, circumscriptions. Do you understand what I mean?

Yes.

From this whole alternating and circumscribing we can abstract sounds or characters, which have often similar meanings in similar contexts. These are quasi our information bits, where it is immediately clear that a bit has meaning only in a context. It is the condensation of a larger context to a smaller context -bit floating freely in the room- which never loses touch with its origin, though. Because if the receiver of the small bit contexts wants to understand us, he must have learned in which larger context they are to be put. What we exchange, therefore, are not bare information, but contexts. 
Although, only to fractions, just bits, since the rest remains relatively unchanged. It has actually been exchanged before - learned.

Interesting. What if we do not talk at all and keep a straight face, but each of us is going to do the opposite? You want to stand up and end the conversation, but I remain seated. Then, how do we come to joint decisions?

How about staring at each other?

Seriously?

Well, it could end up in that. Not as a dominance affectation, but as an unexpressed attunement. Consciously or subconsciously.

You mean telepathically?

Maybe. How should an external observer know this if we do not express it? We could only know as participants. Or not even then if we close our minds too much to the inner expression. However, if the subconscious is not completely different from the conscious - and in this case they could hardly be connected - then we have an i-structured attunement out of mutually alternating awareness.

And how about the bit that we have laid on the table and that is perceived by someone who does not have our context? He reads something else from it, but he reads something. How can he do this at all if everything is context-based?

He might not even read a pure difference, you think?

That's how one can look at it.

That's right; the pure difference is an abstraction, too. If not, it would, indeed, be as concrete as its so-called information carrier, say, a match. Or, for my part, an electron.

Then I ask differently: Why do all electrons look so similar and most of all: How do they make decisions if they are so simple?

Ah, yes, now we could start, of course, with quantum theory, which describes in which patterns such an electron appears, disappears, jumps and curls. These alternations appear to be random, only statistically predictable. But we have to understand that $\mathrm{i}$-structure is much more subtle and complex than electrons or quarks. Because it also encompasses "their" overall combinations and relations, including those of the "observer."

So, I cannot tell you why an electron turns up where it does. But if I am applying our findings on alternation, every electron-like relation will arise from an i-structured attunement process which contains at least elementary free decisions.

This sounds like a subquantum level à la David Bohm plus free will ...

Whether Bohm or not, but sublevel definitely. Even if we would be this sublevel.

Sorry? 
We might even partake of something that causes and dissolves electrons. As long as I don't know anything more specific about this level I cannot locate it.

Okay, we stop speculating. Apparently the "hardness" of the matter perception is the "hard problem" of all idealistic theories. Would you say that your theory is idealistic?

If you insist on this lame division, my theory is, in the end, idealistic. However, it offers a new understanding of consciousness and matter, and it comes to the conclusion that there is ultimately no matter, but everything is consciousness. Rather awareness. Without this understanding one will not accept my explanation of free will, either.

I think so, too. In fact, you put the freedom of choice in the first place, while the stability of the world now requires explanation, rather than the other way around.

Yes, but with the concept of awareness the "hard problem" becomes a "soft problem." Then it is not anymore about the principle, but only about the details to be explored. Such a science of consciousness will be concerned, above all, with direct experiences and reach far beyond today's psychology.

How, nonetheless, do you explain the almost legendary hardness of a billiard ball? According to your idea we would have to fluctuate constantly into it. Why then are we aware of it so stable?

The ball is a ball, of course, only superficially. Instead, its atoms point to many small states of consciousness that we can take up. They are aspects of our awareness. However, they are so distant to our known state of consciousness that we can hardly assign their constant taking up to ourselves. The psychological detour is too big to strike us. Therefore, our deception about an externally "given" ball.

Besides, the alternation between focusses as similar as atoms can be coiled much more densely, so that their common approximation appears massive compared to the loose circumscriptions we have discussed. The possible differences in density are non-linear and hence huge. So they pretend a fundamental difference, which we perceive as hardness.

Could this be the reason for the greater stability of our entity as well? It isn't yet a billiard ball, is $i t ?$

I don't hope so. It unites considerably more varied states and is thus a more dynamic entirety. Its stability must therefore be based on a complex harmony of its alternation, rather than on an adjustment of its phases. In addition, there is the perspectival compression in the funnel stem by our ignorance.

I must think here of dreams. In them, circumscriptions and decisions would have to take place likewise, only much faster.

If we perceive our dreams carefully, and I mean particularly the feelings in them, we learn exactly this: The visually most different scenes are in a feedback-relationship to each other while we make decisions out of these circumscriptions with such ease that they appear to us spontaneous.

But this, they could really be. 
Of course. The subconscious produces suggestions which are being coupled with our dreamconscious judgment and are eventually dismissed or further developed. In every scene there are more points of contact than in the waking state. This great flexibility enables a closer cooperation of the dream state with the subconscious: Decision questions are alternately processed faster.

\section{Perhaps we should allow ourselves more daydreaming.}

Precisely! With our insistence on concentrated alertness we cover too many inspirations. Unless, we don't want any. What I wanted to get at, though, is this: The flexibility in a dream makes us feel the great inner harmony - with some effort even in a nightmare. And the best: This harmony continues in the waking state - then we only perceive it less. If we interpret life like a dream this becomes more conscious to us.

Okay, I have another question: Is a significant degree of freedom of choice dependent on a brain, or at least on a very high complexity?

Basically only on awareness. We have already talked about very simple circumscriptions. For their perception no brain is necessary. We need it for our discussion only. Any little interaction must occur between entireties, thus consist of circumscriptions, and has a certain degree of freedom via its istructure. This freedom cannot be great, indeed; otherwise, these simple structures would dissolve almost immediately and could hardly act as such. In contrast, a complex structure is internally more flexible and just thereby preserves its overall stability. So, it can afford more freedom externally.

As far as it is not restricted by its creator in the broadest sense. Such as for a computer.

That's how it is. The computer's restriction is mainly being not complex, just complicated. We want to be able to control it by rigid programming. Of course, an independently evolving robot is conceivable, with a much more flexible mental center to which we have given only few basic conditions. Whether such a construction leads to a free will distinguishable from occasional crashes remains to be seen.

In a sense we are but again with unknown stability causes - also of matter.

Yet, we have a "material" now from which even stability laws can be constructed: The i-structure or alternation structure pervading anything at all. "Interaction" is only a simplification. "Matter" as well, but it dissolves one floor below.

A stability or symmetry law, such as the conservation of energy, would be then a condensation from a more encompassing alternation structure?

Yes. Alternation is symmetry-oriented anyway, since it just "returns." The condensation of an alternation of potentials to a balance of abstract potential -of pure energy-is therefore not a too long step sequence. But as learned from alternation, there must be alternatives to the conservation of the known energy. Only the "contact surface" for them has been reduced by the condensation.

So, maybe close spiritual spheres could still interfere with physics? 
I'm certain of it. Not only in the quantum field, but everywhere where we do not "look": Between conscious alternation pairs, close to the funnel axis. The perceived energy balance must be thereby disturbed as little as our everyday actions by a second of daydreaming.

The energy conservation is therefore a condensation from a more varied balance of alternating potentials. Potential but is condensed awareness, and awareness is $i$-structured. Hence, both this balance of potentials and the conservation of energy were basically voluntary agreements?

On a much more encompassing level than is conscious to us, yes.

Well, in the face of that, have our actions a noteworthy importance for other individuals? Or are we -hierarchy summit or not-merely sparklets in space?

Since we are all summits of our individual awareness that expands into all other awareness, everything we do in the most comprehensive sense is equivalent! At the top there is always only a single consciousness unit. The perceived difference originates below it -in the larger consciousnessand dissolves again even further below - in the subconscious.

But the consciousness unit is tiny! And if we make the subconscious conscious we restore the conscious difference!

Right. Yet, hierarchy is hierarchy, because they are all infinite. What I decide changes the probabilities in every other awareness and vice versa. Only by persistently fading out large parts of them we can forget their influence or delegate it to others.

Quite concrete: If I want to eliminate poverty in the world, can I do more than making donations and signing petitions?

You are doing more by wanting something different! Even if you do not become active. Because what you think, feel, believe, and wish, enters the focus alternation of your awareness and with it the awareness of all others. They may block the new potential since they decide as freely. But the probability distribution of their alternatives has already changed.

If you really think different, you will soon act differently. This is, however, "only" the visible part, which in turn strengthens the invisible focusing. Altogether, we create a global collective on similar probability lines. More we can hardly expect, since the stability of a situation also has deeper causes in ourselves which we don't want to change at all and which, besides, are usually less conscious to us. So, the difference between poor and rich can be, for example, a thoroughly welcomed challenge - for both sides. Regardless of how we cope with it.

Nevertheless, the knowledge of being an absolutely unique individual among others can induce a shock of loneliness.

Here the knowledge must help that we are even closer connected to each other than we thought, only differently, dynamically. However, some dilute this connection immediately to a diffuse all-is-one consciousness that is certainly more convenient, but merely satisfies an emotional need. 
What do you think in this context of the frequently invoked love?

It depends on what you understand by love. I think you mean neither sex nor infatuation, for both are rather limited, indeed.

And more arbitrary than I would like.

I know what you mean. After all, for many people a long-term need is the basis: They want to maintain and develop the bond. But what do they search beyond the delirious state? A gentler form, Okay. Meanwhile I believe, we should either expand our understanding of love or think beyond this concept. Love is the result of something we would not call so according to current understanding.

What could that be? Possibly your i-structure?

If you consider it unbiasedly, what do you sense?

Utmost abstraction, I would say. And a certain "floating."

Perhaps this is the case only because we are dependent on our thinking that doesn't grasp all aspects. If we, on the other hand, bet only on feelings we have a "blurring." A deeper truth would show up also in the forms of sensation, feeling, and thought. But it would have an own quality, a subtlety and lucidity that satisfies -if it is harmonious- more comprehensively.

Is this the so-called enlightened witness?

I don't know. I don't expect of an enlightened constant equanimity, how boring! But that, even if he gets upset, he knows what he is doing. Not that he controls everything; only that he could if necessary.

I have listened, but I just had to note what occurred to me on the keyword i-structure:

- Alternately identification with me, the other, and the whole

- Mutual enfoldment and unfoldment of the alternation sides

- Existence in the other

- Mirroring of the self

And aren't all these features of love? Whether in relation to people, objects, or All That Is? We can even add the potentials of awareness:

- Freedom

- Harmony

- Value fulfillment

- Creativity

This, too, sounds to me like a circumscription of love. 